ORIGINAL PAPER

\title{
RENAL INTERSTITIAL MAST CELL COUNT IS SIGNIFICANTLY HIGHER IN MEMBRANOPROLIFERATIVE GLOMERULONEPHRITIS THAN IN CLASS IV LUPUS NEPHRITIS
}

\author{
Karolina Kaczmarczyk ${ }^{1}$, Jacek Musiae ${ }^{2}$, Jerzy Soja ${ }^{3}$, Marek Kuźniewsia ${ }^{4}$, \\ Agnieszka Gala-BŁądzińska ${ }^{5}$, Magdalena Biazas ${ }^{1}$, Krzysztof Okoń ${ }^{1}$
}

\begin{abstract}
${ }^{1}$ Department of Pathomorphology, Collegium Medicum, Jagiellonian University, Krakow ${ }^{2}$ Department of Allergy and Immunology, Collegium Medicum, Jagiellonian University, Krakow ${ }^{3}$ Department of Pulmonology, Collegium Medicum, Jagiellonian University, Krakow ${ }^{4}$ Department of Nephrology, Collegium Medicum, Jagiellonian University, Krakow ${ }^{5}$ Department of Internal Medicine, Nephrology and Endocrinology, St Queen Jadwiga District Hospital No. 2 , Rzeszow
\end{abstract}

\section{Introduction}

Systemic lupus erythematosus (SLE) is an autoimmune disease which manifests with the loss of tolerance for self-antigens and the production of autoantibodies. Autoantibodies form immune complexes which accumulate in different organs, leading to inflammation and damage. Systemic lupus erythema- tosus in $50-80 \%$ of cases involves the kidney, causing lupus nephritis (LN), mainly in the form of glomerulonephritis. Glomerular inflammation is followed by the activation of tubular epithelial cells, interstitial fibrosis and eventual renal failure [1].

Glomerular lesions in SLE are classified by the International Society of Nephrology/Renal Pathology Society (ISN/RPS) system or the older World Health 
Organization (WHO) system; LN is divided into six classes with different mesangial, endothelial and epithelial injury patterns. Systemic lupus erythematosus class IV, also called diffuse lupus nephritis, is an endo- or extracapillary glomerulonephritis with diffuse subendothelial immune deposits, with or without mesangial alterations, which can be segmental or global [2].

Membranoproliferative glomerulonephritis (MPGN) is a rare renal disease characterized by diffuse mesangial cell proliferation, along with structural changes in glomerular capillary walls, specifically subendothelial and subepithelial deposits [3, 4]. This pattern is similar to that seen in lupus nephritis ISN/RPS class IV. Membranoproliferative glomerulonephritis can be divided into types I, II (dense deposit disease (DDD)) and III. The relationship between these three subtypes remains uncertain, but DDD is generally regarded as distinct, whereas types I and III are regarded as related entities. Types II and III are rare, while type I shows the highest morphologic similarity to proliferative lupus nephritis; thus from this point onwards, if not otherwise specified, the term MPGN refers to type I only [5].

Mast cells (MCs) are bone marrow-derived cells well known for their contribution to anaphylactic reactions, where they induce inflammation by releasing a range of mediators [6]. They are also known to participate in renal interstitial inflammation, where they contribute to renal fibrosis $[7,8]$. Human mast cells contain granules with two proteases - chymase and tryptase. Mast cells can be divided into two classes, chymase-positive and chymase-negative, due to the fact that only a portion of mast cells are positive for chymase [9-11]. Chymase has the ability to convert angiotensin I to angiotensin II, which regulates intrarenal blood flow, and also participates in renal interstitial fibrosis $[11,12]$. It is involved in renal remodeling by increasing the expression of transforming growth factor $\beta$ (TGF- $\beta$ ) [13]. Trypt-

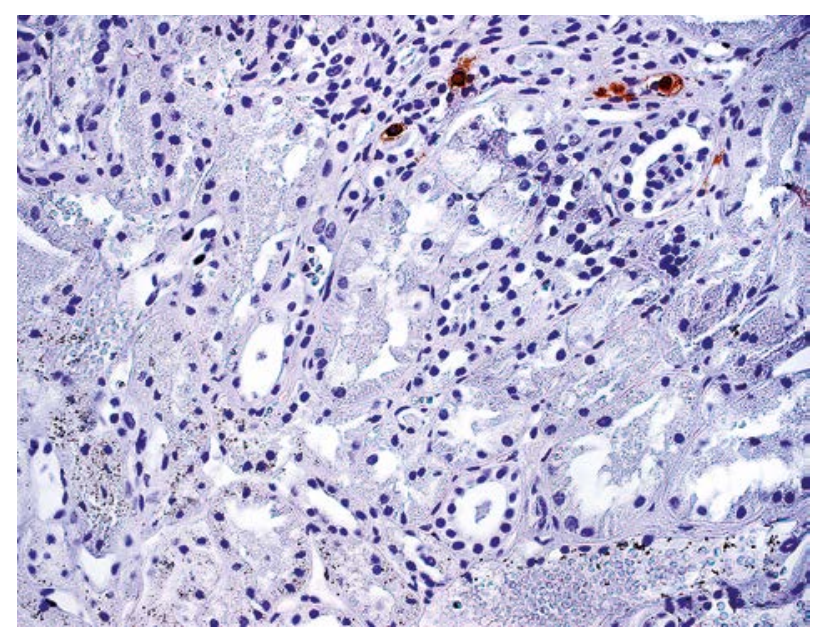

Fig. 1. Chymase positive cells in renal interstitium. Immunohistochemistry, original magnification $400 \times$ ase is a serine protease expressed almost exclusively in mast cells. It has many biological functions, such as its role as a mitogen for fibroblasts, smooth muscle cells and bronchial epithelial cells. Additionally, it has been shown that tryptase participates in skin, lung and renal fibrosis by promoting the proliferation of fibroblasts and the production of extracellular matrix [14]. It is also known for its contribution to acute renal transplant rejection and chronic allograft nephropathy [15].

The aim of the study was to compare the counts of chymase-positive and tryptase-positive mast cells in LN ISN/RPS class IV and MPGN, two different diseases with similar morphologic changes. We examined the relationship between mast cell interstitial infiltration, renal function and diagnosis.

\section{Material and methods}

The material consisted of 61 renal biopsies from 32 patients with LN and 29 patients with MPGN, collected from the archives of the Department of Pathomorphology (Collegium Medicum, Jagiellonian University, Krakow). Only representative biopsies were used for this study.

The material was fixed in formalin, processed by the routine method and embedded in paraffin. $4 \mu \mathrm{m}$ sections were cut from paraffin blocks. Sections were stained with standard hematoxylin and eosin, periodic acid-Schiff, Masson's trichrome, Congo red, and Jones' silver methods for histologic classification.

Primary anti-tryptase antibody (1 : 100; Leica Biosystems $\mathrm{GmbH}$, Wetzlar, Germany) and antichymase antibody (1 : 100; Abcam, Cambridge, UK) were used. The immunohistochemistry was performed according to the routine manual method. The Lab Vision detection system (Thermo Fisher Scientific, Waltham, MA, USA) was used with 3-amino-9-ethylcarbazole as the chromogen. The slides were coun-

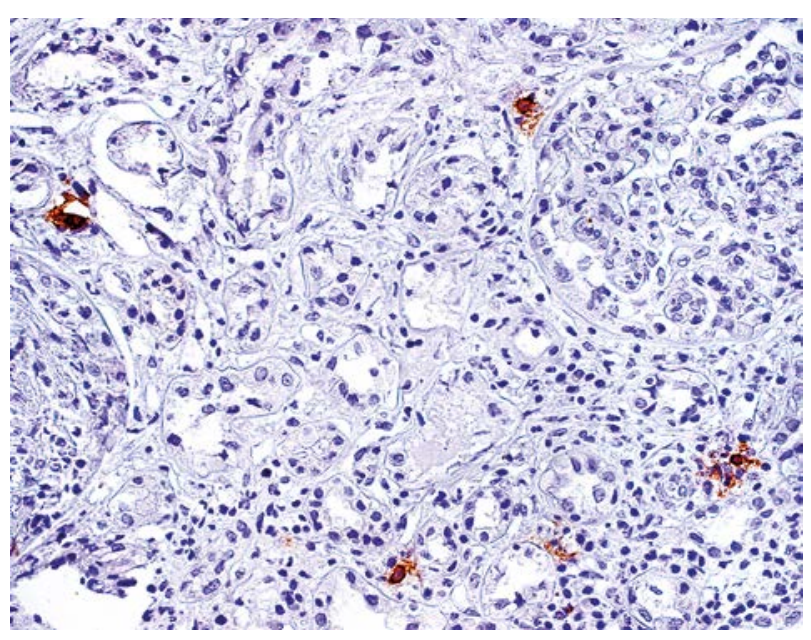

Fig. 2. Tryptase positive cells in renal interstitium. Immunohistochemistry, original magnification $400 \times$ 
terstained with Mayer hematoxylin (Thermo Fisher Scientific) and coverslipped. The immunostained slides were examined on an Olympus CH20 optical microscope equipped with a $40 \times$ lens; the number of immunopositive cells and the number of fields of view were documented. The results were expressed as the sum of positive cells from 10 high power fields, while the area of one high power field was $0.159 \mathrm{~mm}^{2}$. The person performing the assessment was aware of neither the clinical data nor the diagnoses. The values of serum creatinine and daily proteinuria were obtained from the patients' records.

For the assessment of relative interstitial volume (RIV), Masson's trichrome stained sections were used with a Zeiss Standard 25 (Zeiss GmbH, Oberkochen, Germany) microscope equipped with a 121-point rectangular eyepiece grid (Pyser-SGI Ltd., Edenbridge, $\mathrm{UK})$ and a $40 \times$ lens. The well-preserved area of the renal cortex was scanned while noting both the number of fields of view and the number of grid cross sections hitting the renal interstitium. Vessels larger than arterioles and glomeruli were ignored. The results were expressed as the ratio of the points sampled in the interstitium to the total number of points sampled in the renal cortex.

The statistical analysis was performed with Statistica 10 (StatSoft Inc., Tulsa, OK, USA). The Mann-Whitney U test was used, while correlations were measured by Spearman's method. The level of significance was set at 0.05 .

\section{Results}

The mean age of all the patients was $37.52 \pm 13.83$ years (all values expressed as the arithmetic mean $\pm \mathrm{SD}$ ), with a range of 19-69 years. For LN class IV, the mean age was $32.65 \pm 10.53$, while in MPGN it

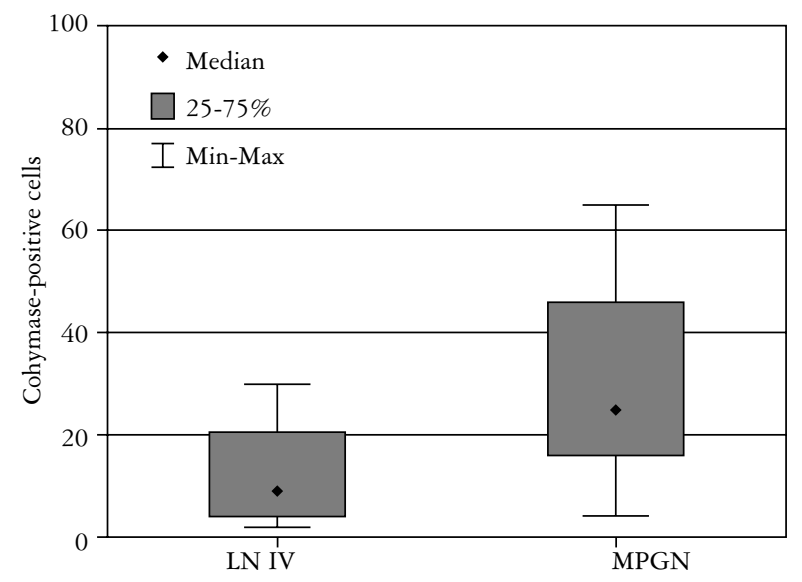

LN IV - lupus nephritis class IV; MPGN - membranoproliferative glomerulonephritis; central point is median; box is $25-75 \%$ range; whiskers are minimummaximum values

Fig. 3. Number of chymase positive cells in lupus nephritis class IV and membranoproliferative glomerulonephritis was $42.9 \pm 15.18$ years. The mean age for females was $35.17 \pm 12.41$ years and $42.35 \pm 15.61$ years for males. These differences were statistically significant.

The mean creatinine serum level was 99.45 $\pm 52.18 \mu \mathrm{mol} / \mathrm{l}$, with a range of $34-310 \mu \mathrm{mol} / \mathrm{l}$. In lupus nephritis class IV, the mean creatinine level was $87.3 \pm 38.53 \mu \mathrm{mol} / \mathrm{l}$, and in MPGN it was 113.84 $\pm 62.51 \mu \mathrm{mol} / \mathrm{l}$. The mean daily proteinuria level was $4.77 \pm 3.19 \mathrm{~g} / \mathrm{d}$, with a range of 0.11 to $14.5 \mathrm{~g} / \mathrm{d}$. In lupus nephritis class IV, the mean daily proteinuria was $4.67 \pm 3.45 \mathrm{~g} / \mathrm{d}$ and in MPGN it was 4.89 $\pm 2.93 \mathrm{~g} / \mathrm{d}$. The differences between the groups were statistically non-significant.

In both groups the mean relative interstitial volume (RIV) was $0.2 \pm 0.09$, with a range of 0.03 to 0.47. In class IV of lupus nephritis, the mean RIV was $0.19 \pm 0.08$ and in MPGN the mean RIV was $0.22 \pm 0.1$. The differences between the groups were statistically non-significant.

The mean count of chymase-positive MCs was $21.94 \pm 22.88$ per 10 high power fields, while the mean count for tryptase-positive MCs was 34.94 \pm 29.29 . The average ratio of chymase-positive to tryptase-positive cells was $0.69 \pm 0.39$.

The differences between groups in chymase-positive and tryptase-positive cell counts were statistically significant $(\mathrm{p}<0.000047$ and $\mathrm{p}<0.000028$, respectively). The differences in the ratio of chymase-positive to tryptase-positive cells between groups were not significant; however, there was a significant negative correlation between the ratio of chymase-positive to tryptase-positive cells and the chymase-positive cell count $(\mathrm{R}=-0.36)$. Chymase-positive MC counts and tryptase-positive MC counts were strongly correlated $(\mathrm{R}=0.8)$. Both chymase-positive MC counts and tryptase-positive MC

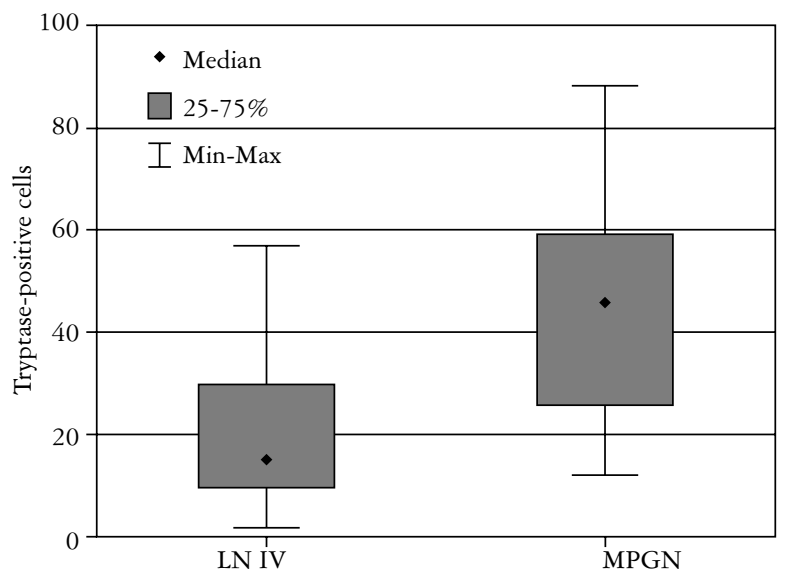

LN IV - lupus nephritis class IV; MPGN - membranoproliferative glomerulonephritis; central point is median; box is $25-75 \%$ range; whiskers are minimum-maximum values

Fig. 4. Number of tryptase positive cells in lupus nephritis class IV and membranoproliferative glomerulonephritis 
counts correlated with RIV $(\mathrm{R}=0.35$ and $\mathrm{R}=0.28$, respectively) and with creatinine level $(\mathrm{R}=0.35$ and $\mathrm{R}=0.43$, respectively). There was also a significant correlation between age and creatinine level and RIV ( $\mathrm{R}=0.28$ and $\mathrm{R}=0.26$, respectively). There was no significant correlation with proteinuria.

\section{Discussion}

Systemic lupus erythematosus class IV and MPGN are two entities differing in etiology and biology, but in some cases they share a similar morphologic pattern. In our study we examined the relationship between the type of renal disease and number of MCs found in the renal tissue. Interestingly, more mast cells were seen in MPGN than in LN class IV, both for chymase-positive and tryptase-positive mast cells. Additionally, the counts of mast cells correlated with relative interstitial volume (RIV), which confirms the role of mast cells in renal interstitial fibrosis.

Membranoproliferative glomerulonephritis displays a pattern of glomerular injury seen in both idiopathic forms and secondary diseases associated with systemic or infectious disorders such as hepatitis $\mathrm{C}$ or LN. The pathogenesis of MPGN is complex and not clearly understood. The pathogenesis is thought to be different in each subtype of MPGN, but the complement system is significantly involved in all types regardless. Type $\mathrm{I}$ is probably due to the accumulation of circulating immune complexes which trigger the complement cascade via the classical pathway. In MPGN type II, the complement system is activated by the alternative pathway, due to the activity of C3 nephritic factor $(\mathrm{C} 3 \mathrm{NeF})$; this IgG autoantibody prevents the inactivation of $\mathrm{C} 3$ convertase $(\mathrm{C} 3 \mathrm{bBb})$ of the alternative pathway, resulting in the breakdown of C3. In type III, the primary role is played by slow-acting nephritic factor $(\mathrm{NeFt})$, which stabilizes the properdin-dependent $\mathrm{C} 5$ convertase $(\mathrm{Cb} 3)$ $2 \mathrm{BbP}$, activating the terminal pathway $[3,4]$. Like in other types of primary glomerulonephritis, the glomerular lesions lead to renal interstitial inflammation and fibrosis, culminating in renal failure [10]. Lupus nephritis is basically an immune complex disease; activation of the complement pathway is also present. In SLE class IV, deposits are located mostly in the subendothelial and mesangial areas, where they activate other renal cells to release proinflammatory mediators, leading to interstitial inflammation and tissue remodeling [16]. Also key to the pathogenesis of SLE is the infiltration of immune cells, consisting of B-cells, T-cells, macrophages, dendritic cells and mast cells [17-22]. The number of mast cells found in the kidney increases in renal diseases, namely diabetic nephropathy, acute renal allograft rejection, IgA nephropathy, amyloid, renovascular ischemia, reflux nephropathy, polycystic kidney disease and drug-induced nephropathy $[7,10]$.

Little information is available with regards to the role of mast cells in LN and membranoproliferative glomerulonephritis and to the contribution of these cells to the pathogenesis of these diseases.

Togawa $e t$ al. investigated chymase expression in renal biopsies from children with inter alia MPGN. The study showed that the number of chymase-positive mast cells correlates with the mean intensity of the interstitial fibrosis, which is similar to the results obtained in our study [12].

Danilewicz et al. analyzed the relationship between mast cell count and the expression of interstitial alpha-smooth muscle actin (SMA), along with the interstitial inflammatory infiltrates in MPGN type I. They found significant positive correlations between interstitial tryptase positive cells and interstitial expression of SMA, interstitial volume, serum creatinine as well as $\mathrm{CD} 43+$ and $C D 68+$ cells, which suggests the role of MCs in the development of interstitial fibrosis [23]. The same group conducted an analogous study with rebiopsied patients with MPGN, where they obtained similar results [24]. Danilewicz et al. demonstrated in another study that the number of interstitial tryptase-positive cells was significantly higher in class $\mathrm{V}$ lupus nephritis than in idiopathic membranous glomerulopathy. Both groups exhibited significant positive correlations between mast cell count and relative interstitial volume (RIV), along with serum creatinine level [25]. In our study, more mast cells were seen in MPGN than in LN. In our present and previous studies [26], we have found the same positive correlation with RIV and creatinine level. Hiromura et al. examined the number of interstitial mast cells in both MPGN and LN. They found a correlation between mast cell count, interstitial fibrosis, creatinine level and leukocyte infiltration, but not with urinary protein excretion; the same results were obtained in our study. In our previous study, we found that mast cell counts differ across classes of SLE, which suggests that these cells play a role in the pathogenesis of LN [26].

We have shown that despite the similar pattern of pathological changes in the renal glomerulus in $\mathrm{LN}$ class IV and MPGN, the quantity of mast cells in the renal interstitium is different. We also confirmed that mast cells contribute to the tubulointerstitial fibrosis, while their numbers correlate with RIV. This necessitates further investigation to explain the role of mast cells in the pathogenesis of and MPGN.

The authors declare no conflict of interest.

The publication of this article was supported by the Faculty of Medicine, Jagiellonian University Medical College, Leading National Research Centre (KNOW) 20122017. 
The authors would like to thank Mr. Ian B. Perera for bis assistance in editing the manuscript and Mr. Krzysztof Skomski for preparing the microphotographs.

\section{References}

1. Hałoń A. Kidney changes in systemic lupus erythematosus and other autoimmune diseases. Pol J Pathol 2011; 62: 57-71.

2. Weening JJ, D'Agati VD, Schwartz MM, et al. The classification of glomerulonephritis in systemic lupus erythematosus revisited. Kidney Int 2004; 65: 521-530.

3. Alchi B, Jayne D. Membranoproliferative glomerulonephritis Pediatr Nephrol 2010; 25: 1409-1418.

4. Nakopoulou L. Membranoproliferative glomerulonephritis Nephrol Dial Transplant 2001; 16: 71-73.

5. Okon K. Glomerular kidney disease that is associated with nephrotic syndrome or rapidly advancing renal failure. Pol J Pathol 2011; 1: s48-56.

6. Galli SJ, Wershil BK. The two faces of the mast cell. Nature 1996; 381: 21-22.

7. Hiromura K, Kurosawa M, Yano S, et al. Tubulointerstitial mast cell infiltration in glomerulonephritis. Am J Kidney Dis 1998; 32: 593-599.

8. Holdsworth SR, Summers SA. Role of mast cells in progressive renal diseases. J Am Soc Nephrol 2008; 19: 2254-2261.

9. Buckley MG, McEuen AR, Walls AF. The detection of mast cell subpopulations in formalin-fixed human tissues using a new monoclonal antibody specific for chymase. J Pathol 1999, 189: 138-143.

10. Okoń K, Stachura J. Increased mast cell density in renal interstitium is correlated with relative interstitial volume, serum creatinine and urea especially in diabetic nephropathy but also in primary glomerulonephritis. Pol J Pathol 2007; 58: 193-197.

11. Andoh A, Deguchi Y, Inatomi O, et al. Immunohistochemical study of chymase-positive mast cells in inflammatory bowel disease. Oncol Rep 2006; 16: 103-107.

12. Togawa H, Nakanishi K, Shima Y, et al. Increased chymase-positive mast cells in children with crescentic glomerulonephritis. Pediatr Nephrol 2009; 24: 1071-1075.

13. Wasse $\mathrm{H}, \mathrm{Naqvi} \mathrm{N}$, Husain A. Impact of mast cell chymase on renal disease progression. Curr Hypertens Rev 2012; 8: 15-23.

14. Kondo S, Kagami S, Kido H, et al. Role of mast cell tryptase in renal interstitial fibrosis. J Am Soc Nephrol 2001; 12: 1668 1676.

15. Barczyk M, Mysliwiec M, Kalinowski M, et al. Mast cells tryptase in patients after renal transplantation. Transplant Proc. 2008; 40: 3437-3439.

16. Lech M, Anders HJ. The pathogenesis of lupus nephritis. J Am Soc Nephrol. 2013; 24: 1357-1366.

17. Steinmetz OM, Velden J, Kneissler U, et al. Analysis and classification of B-cell infiltrates in lupus and ANCA-associated nephritis. Kidney Int 2008; 74: 448-457.

18. Clatworthy MR, Smith KG. B cells in glomerulonephritis: focus on lupus nephritis. Semin Immunopathol 2007; 29: 337-353.

19. Fiore N, Castellano G, Blasi A, et al. Immature myeloid and plasmacytoid dendritic cells infiltrate renal tubulointerstitium in patients with lupus nephritis. Mol Immunol 2008; 45: 259-265.

20. Castellano G, Trouw LA, Fiore N, et al. Infiltrating dendritic cells contribute to local synthesis of $\mathrm{C} 1 \mathrm{q}$ in murine and human lupus nephritis. Mol Immunol 2010; 47: 2129-2137.

21. Teichmann LL, Ols ML, Kashgarian M, et al. Dendritic cells in lupus are not required for activation of $\mathrm{T}$ and $\mathrm{B}$ cells but promote their expansion, resulting in tissue damage. Immunity 2010; 33: 967-978.

22. Tucci M, Stucci S, Strippoli S, et al. Cytokine overproduction, T-cell activation, and defective T-regulatory functions promote nephritis in systemic lupus erythematosus. J Biomed Biotechnol. 2010; 2010: 457146.
23. Danilewicz M, Wągrowska-Danilewicz M. Quantitative analysis of the interstitial mast cells in idiopathic mesangiocapillary glomerulonephritis type I. Nefrologia 2001; 21: 253-259.

24. Danilewicz M, Wągrowska-Danilewicz M. Immunohistochemical analysis of the interstitial mast cells in rebiopsied patients with idiopathic mesangial proliferative glomerulonephritis. Pol J Pathol 2005; 56: 63-68.

25. Danilewicz M, Wagrowska-Danilewicz M. Quantitative analysis of interstitial mast cells in lupus and non-lupus membranous glomerulopathy. Pol J Pathol 2001; 52: 211-217.

26. Kaczmarczyk K, Kosalka J, Soja J, et al. Renal interstitial mast cell counts differ across classes of proliferative lupus nephritis. Folia Histochem Cytobiol 2014; 52: 218-224.

\section{Address for correspondence}

\section{Krzysztof Okoń MD PhD}

Department of Pathomorphology

Jagiellonian University Collegium Medicum

Grzegórzecka 16

31-531 Krakow, Poland

e-mail: mpokon@cyf-kr.edu.pl 